\title{
The dynamics of a fully radiative rapidly rotating star enclosed within a spherical box
}

\author{
F. Espinosa Lara and M. Rieutord
}

\author{
Laboratoire d'Astrophysique de Toulouse et Tarbes, UMR 5572, CNRS et Université Paul Sabatier Toulouse 3, 14 avenue E. Belin, \\ 31400 Toulouse, France \\ e-mail: rieutord@ast.obs-mip.fr
}

Received 9 February 2007 / Accepted 22 April 2007

\begin{abstract}
Context. Recent results from interferometry and asteroseismology require models of rapidly rotating stars that are more and more precise.

Aims. We describe the basic structure and the hydrodynamics of a fully radiative star as a preliminary step towards more realistic models of rotating stars.

Methods. We consider a solar mass of perfect gas enclosed in a spherical container. The gas is self-gravitating and rotating, and is the seat of nuclear heating, and heat diffusion is due to radiative diffusion with Kramers type opacities. Equations are solved numerically with spectral methods in two dimensions with a radial Gauss-Lobatto grid and spherical harmonics.

Results. We computed the centrifugally flattened structure of such a star: the von Zeipel model, which says that the energy flux is proportional to the local effective gravity is tested. We show that it overestimates the ratio of the polar to the equatorial energy flux by almost a factor 2. We also determine the Brunt-Väisälä frequency distribution and show that outer equatorial regions in a radiative zone are convectively unstable when the rotation is fast enough. We compute the differential rotation and meridional circulation stemming from the baroclinicity of the star and show that, in such radiative zones, equatorial regions rotate faster than polar ones. The surface differential rotation is also shown to reach a universal profile when rotation is slow enough (less than $36 \%$ of the breakup one), as long as viscosity and Prandlt number remain small.
\end{abstract}

Key words. hydrodynamics - stars: rotation

\section{Introduction}

Recent observations of nearby stars with optical or infrared interferometers have opened a new window on rapidly rotating stars. The work of Domiciano de Souza et al. (2005); Zorec et al. (2005); McAlister et al. (2005); Peterson et al. (2006a); Peterson et al. (2006b); Kervella \& Domiciano de Souza (2006) and Aufdenberg et al. (2006) show that observational techniques are now able to constrain the surface distribution of the energy flux of these stars along with their precise shape on the background sky. Data are usually fit by a simple brightness law derived from a Roche-von Zeipel model (e.g. Domiciano de Souza et al. 2002) where it is assumed that the shape of the star is determined by a Roche model (all the mass is concentrated in the center), while the brightness distribution obeys the von Zeipel law, namely the energy flux is proportional to local effective gravity. If such a model is efficient at giving a first interpretation of the observation and especially getting an idea of the orientation of the stellar rotation axis, as well as its angular velocity, one now wishes to go beyond this simple model. This step is difficult, however, as it means building a self-consistent two-dimensional model of a star and then being able to make it evolve till the assumed age of the observed object.

Many attempts have been made to reach this goal, but most of them have not gone beyond the construction of barotropic stars without any dynamics or evolution (see the recent work of Roxburgh 2004 and Jackson et al. 2005). The inclusion of fluid dynamics is, however, important for the models to be physically self-consistent. Rotating stars are indeed baroclinic, and fluid flows are present everywhere, even in radiative zones. Their long-term effect is the mixing of elements and the transport of angular momentum (see Zahn 1992), so they cannot be ignored in the evolution of rotating stars.

Previous work on models of baroclinic stars is rare. Only Uryu \& Eriguchi $(1994,1995)$ constructed global 2D models of rapidly rotating stars with baroclinicity, but their neglect of viscosity removed any meridional circulation and left differential rotation undetermined. More recently, Rieutord (2006) used a Boussinesq model (i.e. a nearly incompressible fluid) for investigating the baroclinic flows on a global scale. In this case, it could be shown how viscosity determines the differential rotation and that this rotation strongly depends on the Prandtl number.

The present work aims at describing a more realistic, physically self-consistent, approach of a rotating, fully radiative star. Of course this is a model and real physics is still very simplified, but no arbitrary assumption needs to be made. In a few words, our model can be described as follows: we consider a perfect gas contained in a rigid sphere; the gas is rotating and self-gravitating; the mass inside the sphere is high enough so that the central temperature and density permit nuclear reactions. The gas is a viscous fluid conducting heat through radiative diffusivity derived from Kramers type opacities. The bounding sphere allows us to impose simple (but physically relevant) boundary conditions on the gravitational potential, temperature, pressure, and velocity field. Roughly speaking, these conditions specify (i) that the gravitational potential connects to the one pervading vacuum, (ii) that temperature connects to the temperature field 
of a medium with a constant absorption coefficient radiating like a black body, (iii) that some radial stress can be accepted by the bounding sphere, but (iv) that matter slips freely and tangentially on the sphere. This set-up, which is similar to a laboratory experiment, simplifies the numerical resolution of the equations by allowing the use of spherical coordinates while the distribution of mass is not spherically symmetric. Fortunately, the bulk properties of this "star" do not depend very much on the physics imposed at the outer boundary. The next step will be to replace the sphere by a spheroid that fits an isobar perfectly; however, this step, which needs to use spheroidal coordinates with multidomains, is numerically challenging and can be undertaken only on a physically understood system.

We have voluntarily removed the convection zones that need a generalization of the mixing-length theory in two dimensions including the effects of rotation. Here we focus on a rotating radiative star and examine the dynamical properties of its baroclinic state.

The paper is organized as follows. We next describe our model (Sect. 2) and the numerical methods we use for solving the equations (Sect. 3). We then describe some tests of the model showing internal accuracy and how they compare to onedimensional models (Sect. 4). In Sect. 5 results are presented and discussed, and conclusions follow.

\section{The model}

We consider a viscous, self-gravitating fluid rotating with a mean angular velocity $\boldsymbol{\Omega}=\boldsymbol{\Omega} \boldsymbol{e}_{z}$. The fluid is supposed to be inside an immutable sphere of radius $R$, so the outer boundary can be taken at $r=R$. This simplifies the problem by allowing spherical coordinates to represent the variables, although the external boundary does not fit an isobar. tions

In a steady configuration, the fluid is governed by the equa-

$$
\left\{\begin{array}{l}
\Delta \phi=4 \pi G \rho \\
\rho T \boldsymbol{v} \cdot \nabla s=-\nabla \cdot \boldsymbol{F}+\varepsilon \\
\rho(2 \boldsymbol{\Omega} \times \boldsymbol{v}+\boldsymbol{v} \cdot \nabla \boldsymbol{v})=-\nabla P-\rho \nabla\left(\phi-\frac{1}{2} \Omega^{2} r^{2} \sin ^{2} \theta\right)+\boldsymbol{F}_{v} \\
\boldsymbol{\nabla} \cdot(\rho \boldsymbol{v})=0
\end{array}\right.
$$

where $\phi$ is the gravitational potential, $\rho$ the density, $s$ the specific entropy, $T$ the temperature, $\boldsymbol{v}$ the fluid velocity with respect to a frame rotating at angular velocity $\boldsymbol{\Omega}$. Also, $P$ is the pressure and $\varepsilon$ the nuclear energy generation rate per unit volume. For a compressible fluid with constant dynamical viscosity $\mu$, the viscous force can be written as

$\boldsymbol{F}_{v}=\mu\left(\Delta \boldsymbol{v}+\frac{1}{3} \nabla(\boldsymbol{\nabla} \cdot \boldsymbol{v})\right)$

For the energy flux, we will consider only radiative transport,

$\boldsymbol{F}=-\chi \nabla T$

where $\chi(\rho, T)$ is the radiative diffusivity. The equation of state is that of an ideal gas mixed with photons; hence, we use

$p=\mathcal{R}_{M} \rho T+\frac{a}{3} T^{4}$

where $\mathcal{R}_{M}$ is the gas constant divided by the mean molecular weight and $a$ the radiation density constant.

\subsection{Stellar microphysics}

We assume that the fluid is in the conditions of a stellar plasma typical of the sun's radiative zone. The nuclear energy generation rate per unit volume has the form

$\varepsilon=\varepsilon_{0} X^{2} \rho^{2} T^{-2 / 3} \mathrm{e}^{-b T^{-1 / 3}}$

where $X$ is the hydrogen mass fraction. For the constants $\varepsilon_{0}$ and $b$ we adopt the values of the CESAM code (Morel 1997), namely

$b=3600$ and $\varepsilon_{0}=8.37 \times 10^{10} \quad(\mathrm{cgs})$

for the pp-chain.

In order to describe the radiative transport of energy, we use the opacity given by power laws:

$\kappa=\kappa_{0} T^{-\beta} \rho^{\eta}$,

so that the radiative diffusivity can be written as

$\chi=\frac{16 \sigma T^{3}}{3 \kappa \rho}=\chi_{0} T^{\beta+3} \rho^{-\eta-1}$.

In particular we use $\beta=1.97541, \eta=0.138316$, and $\kappa_{0}=7.1548412 \times 10^{13} \mathrm{cgs}$, following some fitting formulae ${ }^{1}$ proposed by J. Christensen-Dalsgaard for simple solar models (e.g. Christensen-Dalsgaard \& Reiter 1995).

\subsection{Boundary conditions}

In order to solve the full system of equations, we must complete it with boundary conditions. At the center of the star $(r=0)$ we just need to impose regularity on the solutions. At the outer boundary, i.e. on the rigid bounding sphere, we impose that the fluid slips freely and thus use stress-free boundary conditions. Hence, we have

$u_{r}=\sigma_{r \theta}=\sigma_{r \varphi}=0 \quad$ at $\quad r=R$

where $\sigma_{r \theta}$ and $\sigma_{r \varphi}$ are the horizontal components of the stress on the outer sphere.

Since the sphere is rigid, it can support some normal stress, and pressure is not constant on it any more than the density and temperature. As the true surface of the star is outside the box, pressure needs to be fixed somewhere. We thus impose the polar pressure. This condition imposes the place where our container "cuts" the star envelope.

The usual boundary conditions on the temperature are that the boundary radiates like a black body. We thus write

$-\chi \frac{\partial T}{\partial r}=\sigma T^{4} \quad$ at $\quad r=R$.

Using the expression of radiative diffusivity, this condition is equivalent to

$\frac{\partial T}{\partial r}+\sigma_{T} T=0$

where $\sigma_{T}=3 \rho \kappa / 16$. Obviously, $\sigma_{T}$ depends on latitude, but to avoid unnecessary complications (nonlinearities) we take it as a constant. Physically, this means that at the boundary the fluid meets a medium with constant absorption coefficient radiating like a black body.

Finally, the boundary conditions on the gravitational potential are that it matches the vacuum solution, which vanishes at infinity.

1 The proposed formula is $\frac{1}{\kappa}=\frac{1}{\kappa_{i}}+\frac{1}{\kappa_{e}}$ with $\kappa_{i}$ as in (7) and $\kappa_{e}=$ $1.6236784 \times 10^{-33} \rho^{0.407895} T^{9.28289}$; we use only the "interior" opacity, leaving the "envelope" one. 


\subsection{Dimensionless equations}

We scale the physical quantities, so that the solutions are of order unity. For that, we choose the free-fall time scale $\lambda=\left(4 \pi G \rho_{\mathrm{c}}\right)^{-1 / 2}$, the radius of the container $R$ as the length scale, the central values of temperature $T_{\mathrm{c}}$ and density $\rho_{\mathrm{c}}$ for the corresponding scales, and the gas constant $\mathcal{R}_{M}$ for the entropy scale. With dimensionless variables, the equations are now written as

$$
\left\{\begin{array}{l}
\Delta \phi=\rho \\
\frac{\mathcal{P}}{E} \rho T \boldsymbol{u} \cdot \nabla s=\nabla \cdot(\chi \nabla T)+\Lambda \varepsilon \\
\rho(2 \boldsymbol{\Omega} \times \boldsymbol{u}+\boldsymbol{u} \cdot \nabla \boldsymbol{u})=-\nabla p-\rho \nabla \phi_{\mathrm{eff}}+E \boldsymbol{F}_{u} \\
\boldsymbol{\nabla} \cdot(\rho \boldsymbol{u})=0 \\
p=\pi_{\mathrm{c}}\left(\rho T+\frac{1-\beta_{\mathrm{c}}}{\beta_{\mathrm{c}}} T^{4}\right)
\end{array}\right.
$$

where $\phi_{\mathrm{eff}}=\phi-\frac{1}{2} \Omega^{2} r^{2} \sin ^{2} \theta$ and $\boldsymbol{F}_{u}=\Delta \boldsymbol{u}+\frac{1}{3} \nabla(\boldsymbol{\nabla} \cdot \boldsymbol{u})$. We also introduced the dimensionless numbers

$E=\frac{\mu \lambda}{\rho_{\mathrm{c}} R^{2}}, \quad \mathcal{P}=\frac{\mu \mathcal{R}_{M}}{\chi_{\mathrm{c}}}$.

Here, $E$ is a kind of Ekman number associated with the limit angular velocity $\sqrt{4 \pi G \rho_{\mathrm{c}}}$, and $\mathcal{P}$ the Prandtl number at the center. The Ekman number measures the importance of viscous force relative to Coriolis force, and its true definition is $E / \Omega$; here we had to keep out the non-dimensional rotation rate for convenience. In stellar conditions $E / \Omega \ll 1$. Other, more stellar, dimensionless numbers have been introduced:

$\pi_{\mathrm{c}}=\frac{\mathcal{R}_{M} T_{\mathrm{c}} \lambda^{2}}{R^{2}}, \quad \Lambda=\frac{\varepsilon_{\mathrm{c}} R^{2}}{\chi_{\mathrm{c}} T_{\mathrm{c}}} \quad$ and $\quad \beta_{\mathrm{c}}=\frac{\pi_{\mathrm{c}}}{\pi_{\mathrm{c}}+\frac{a T_{\mathrm{c}} \lambda^{2}}{3 \rho_{\mathrm{c}} R^{2}}}$,

where $\pi_{\mathrm{c}}$ is the dimensionless central gas pressure and $\beta_{\mathrm{c}}$ the usual ratio of gas to total pressure at the star center. Once we fix the microphysics of the problem, the dimensionless numbers $\pi_{\mathrm{c}}$ and $\Lambda$ should appear as part of the solution, as they determine the central temperature and density (or mass) of the configuration.

The quantities $\chi$ and $\varepsilon$ have been scaled using their central values $\chi_{\mathrm{c}}$ and $\varepsilon_{\mathrm{c}}$, so that the dimensionless radiative diffusivity and energy generation rate are given by

$\chi=T^{\beta+3} \rho^{-\eta-1}$ and $\varepsilon=\rho^{2} T^{-2 / 3} \mathrm{e}^{-\alpha\left(T^{-1 / 3}-1\right)}$

where $\alpha=b T_{\mathrm{c}}^{-1 / 3}$. Using these expressions and writing the entropy gradient as a function of the temperature and pressure gradients, the equation of energy balance reads

$\Delta T+\nabla \ln \chi \cdot \nabla T-\frac{\mathcal{P}}{E} \boldsymbol{a} \cdot \boldsymbol{u}=-\Lambda \frac{\rho^{\eta+3}}{T^{\beta+11 / 3}} \mathrm{e}^{-\alpha\left(T^{-1 / 3}-1\right)}$

where we introduced the vector

$\boldsymbol{a}=\frac{\rho^{\eta+1}}{T^{\beta+3}}\left(\frac{\gamma}{\gamma-1} \rho \nabla T-\frac{1}{\pi_{\mathrm{c}}} \nabla p\right)$

which makes explicit the entropy gradient; $\gamma$ is the adiabatic exponent.

The scaling of the velocity field requires some consideration. In Rieutord (2006) it is shown that the meridional circulation, i.e. the radial and meridional components of the velocity field, is proportional to the Ekman number; therefore, we write:

$\boldsymbol{u}=E u_{r} \boldsymbol{e}_{r}+E u_{\theta} \boldsymbol{e}_{\theta}+u_{\varphi} \boldsymbol{e}_{\varphi}$.

The system (10) is the set of nonlinear PDE that governs the radiative zone of a rotating star when no turbulence or magnetic field troubles the place. It is this system that will give us the first view of the dynamics inside the central parts of a rapidly rotating fully radiative star.

\section{Numerical method}

One-dimensional stellar evolution codes usually use a few thousand of grid points to model and follow the evolution of a star. With the same kind of discretization (i.e. finite differences), the number of grid points would grow by a factor of a few hundred for a two-dimensional model. This large number of points is certainly very heavy to manage; two-dimensional models require more efficient discretization, which is why we adopted spectral methods.

Spectral methods are best-known for their precision when the number of grid points is limited (Peyret 2002; Grandclément 2006; Bonazzola et al. 1999). On the dark side, they have never been used in stellar modeling (to our knowledge) and are rather "touchy" as far as stability is concerned; more particularly, discontinuities require special care like a multi-domain approach does. Nevertheless, the precision they can achieve is a major advantage, especially when the oscillation spectrum of a star needs to be computed. The advances in observational asteroseismology, with space missions like COROT, indeed require very precise models (Reese et al. 2006).

The discretization of the equations is made via an expansion of the unknowns onto the spherical harmonic basis for the angular part and using Gauss-Lobatto collocation nodes for the radial part. This latter grid is associated with Chebyshev polynomials (e.g. Canuto et al. 2006; Fornberg 1998).

\subsection{Projection on the spherical harmonics}

The variables are thus first projected on the spherical harmonics. Hence, for the scalar quantities:

$f=\sum f_{l}(r) Y_{l}^{0}(\theta)$.

Note that, due to the symmetry of the problem, solutions are axisymmetric and therefore $m=0$. They are also symmetric with respect to the equatorial plane, so scalar quantities need only spherical harmonics of an even order. For the velocity, we follow Rieutord (1987) and use the expansion:

$\boldsymbol{u}=\sum_{l=0}^{L_{\max }} E u_{l}(r) \boldsymbol{R}_{l}^{0}+E v_{l}(r) \boldsymbol{S}_{l}^{0}+w_{l}(r) \boldsymbol{T}_{l}^{0}$,

where the vectorial spherical harmonics are

$\boldsymbol{R}_{l}^{m}=Y_{l}^{m} \boldsymbol{e}_{r}$

$\boldsymbol{S}_{l}^{m}=\frac{\partial Y_{l}^{m}}{\partial \theta} \boldsymbol{e}_{\theta}+\frac{1}{\sin \theta} \frac{\partial Y_{l}^{m}}{\partial \varphi} \boldsymbol{e}_{\varphi}$

$\boldsymbol{T}_{l}^{m}=\frac{1}{\sin \theta} \frac{\partial Y_{l}^{m}}{\partial \varphi} \boldsymbol{e}_{\theta}-\frac{\partial Y_{l}^{m}}{\partial \theta} \boldsymbol{e}_{\varphi}$.

The projection of the momentum equation yields three equations:

$$
\begin{array}{r}
\frac{\mathrm{d} p_{l}}{\mathrm{~d} r}+2\langle\rho\rangle \Omega\left[(l-1) \alpha_{l} w_{l-1}-(l+2) \alpha_{l+1} w_{l+1}\right] \\
-E^{2}\left[\frac{4}{3} \frac{\mathrm{d}^{2} u_{l}}{\mathrm{~d} r^{2}}+\frac{8}{3 r} \frac{\mathrm{d} u_{l}}{\mathrm{~d} r}-\frac{(l(l+1)+8 / 3)}{r^{2}} u_{l}\right. \\
\left.-\frac{l(l+1)}{3 r} \frac{\mathrm{d} v_{l}}{\mathrm{~d} r}+\frac{7 l(l+1)}{3 r^{2}} v_{l}\right]=\mathrm{rhsu}_{l}
\end{array}
$$

$$
\begin{aligned}
& \frac{p_{l}}{r}+2\langle\rho\rangle \Omega\left[\frac{l-1}{l} \alpha_{l} w_{l-1}+\frac{l+2}{l+1} \alpha_{l+1} w_{l+1}\right] \\
& -E^{2}\left[\frac{\mathrm{d}^{2} v_{l}}{\mathrm{~d} r^{2}}+\frac{2}{r} \frac{\mathrm{d} v_{l}}{\mathrm{~d} r}-\frac{4 l(l+1)}{3 r^{2}} v_{l}+\frac{1}{3 r} \frac{\mathrm{d} u_{l}}{\mathrm{~d} r}+\frac{8}{3 r^{2}} u_{l}\right]=\operatorname{rhsv}_{l}
\end{aligned}
$$


Table 1. Non-linear terms that appear in the right-hand sides of the equations of motion (18-20), (24) and (25).

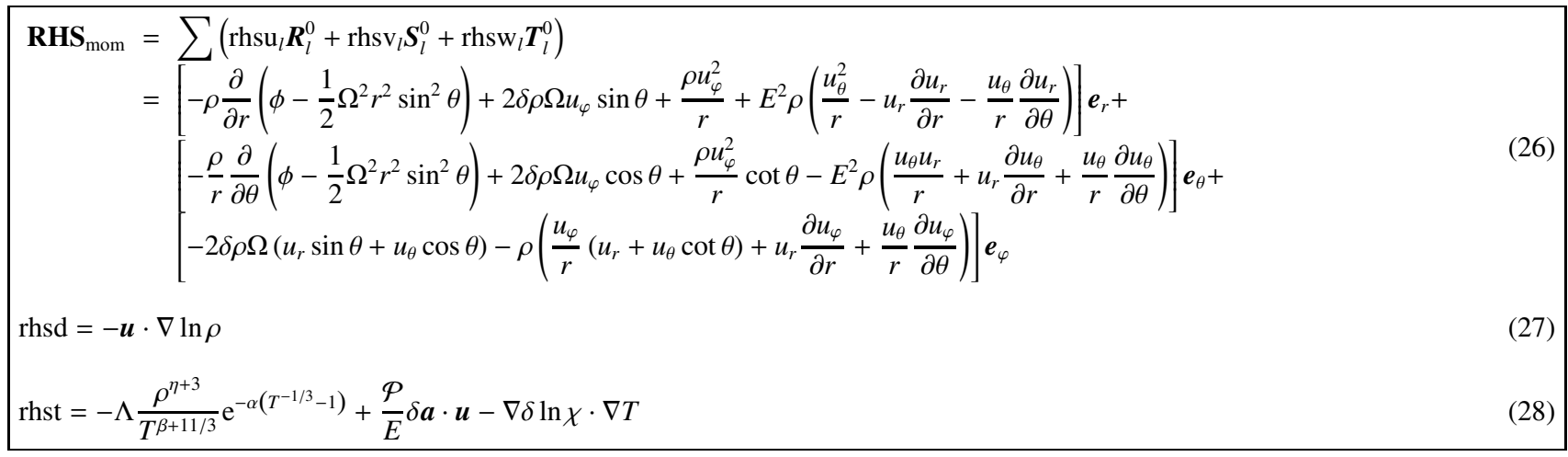

$$
\begin{array}{r}
2\langle\rho\rangle \Omega\left[\frac{\alpha_{l}}{l} u_{l-1}-\frac{\alpha_{l+1}}{l+1} u_{l+1}-\frac{l-1}{l} \alpha_{l} v_{l-1}-\frac{l+2}{l+1} \alpha_{l+1} v_{l+1}\right] \\
-\left[\frac{\mathrm{d}^{2} w_{l}}{\mathrm{~d} r^{2}}+\frac{2}{r} \frac{\mathrm{d} w_{l}}{\mathrm{~d} r}-\frac{l(l+1)}{r^{2}} w_{l}\right]=\mathrm{rhsw}_{l},
\end{array}
$$

where we have introduced the spherically averaged quantities

$$
\langle f\rangle=\frac{1}{4 \pi} \int_{4 \pi} f \mathrm{~d} \Omega=\frac{f_{0}(r)}{\sqrt{4 \pi}}
$$

and the coupling coefficient

$\alpha_{l}=\frac{l}{\sqrt{4 l^{2}-1}}$.

The other equations yield

$$
\begin{aligned}
& \frac{\mathrm{d}^{2} \phi_{l}}{\mathrm{~d} r^{2}}+\frac{2}{r} \frac{\mathrm{d} \phi_{l}}{\mathrm{~d} r}-\frac{l(l+1)}{r^{2}} \phi_{l}=\rho_{l} \\
& \frac{\mathrm{d} u_{l}}{\mathrm{~d} r}+\frac{2 u_{l}}{r}-\frac{l(l+1)}{r^{2}} v_{l}=\operatorname{rhsd}_{l} \\
& \frac{\mathrm{d}^{2} T_{l}}{\mathrm{~d} r^{2}}+\left(\frac{2}{r}+\frac{\mathrm{d}}{\mathrm{d} r}\langle\ln \chi\rangle\right) \frac{\mathrm{d} T_{l}}{\mathrm{~d} r}-\frac{l(l+1)}{r^{2}} T_{l}-\mathcal{P}\left\langle a_{r}\right\rangle u_{l}=\text { rhst }_{l} .
\end{aligned}
$$

The right-hand terms are essentially non-linear terms that are gathered in Table 1 . In their expressions $\delta$ denotes the nonspherical part of a variable, i.e. $\delta f=f-\langle f\rangle$.

\subsection{Boundary conditions}

Boundary conditions have to be expressed on the radial functions. Regularity of the solutions at the star center demands the regularity of the radial functions at $r=0$. As far as the pressure is concerned, we fix the polar value to $p_{\mathrm{s}}$ which fixes the constant needed by the solution of the momentum equation. Stress-free boundary conditions on the velocity field give (Rieutord 1987)

$$
\left\{\begin{array}{l}
u_{l}=0 \\
r \frac{\mathrm{d} v_{l}}{\mathrm{~d} r}-v_{l}=0 \\
r \frac{\mathrm{d} w_{l}}{\mathrm{~d} r}-w_{l}=0
\end{array}\right.
$$

at the surface. The gravitational potential at the surface matches that of the vacuum

$$
r \frac{\mathrm{d} \phi_{l}}{\mathrm{~d} r}+(l+1) \phi_{l}=0 \quad \text { at } \quad r=1
$$

Finally, the radial functions of the temperature verify

$r \frac{\mathrm{d} T_{l}}{\mathrm{~d} r}+\sigma_{T} T_{l}=0$

at the surface.

\subsection{Algorithm for iterations}

Equations are solved iteratively because of nonlinearities. We follow and generalize the algorithm used by Bonazzola et al. (1998) for polytropic stellar models. The idea is to set the equations in the form

$\mathcal{L}_{n}\left(\boldsymbol{y}_{n+1}\right)=R H S\left(\boldsymbol{y}_{n}, \boldsymbol{y}_{n-1}\right)$

where $\mathcal{L}_{n}$ is a linear operator that may depend on the n-th iterate. Such a scheme is also known as the fixed-point algorithm. Convergence depends on the norm of the linear operator and the closeness to the solution; the dependence of RHS on $\boldsymbol{y}_{n-1}$ stands for the relaxation terms. Unlike Henyey's method, which is a Newton type algorithm, this scheme does not need the computation of the Jacobian matrix, which is quite convenient with our discretization and high order operators.

\section{Tests of the model}

\subsection{Internal accuracy}

In Fig. 1 we show the spectra of the different physical quantities. As these spectra are scaled by the maximum value, they show the internal relative precision of the solutions due to truncation. In this example, spectral convergence is very good on the gravitational potential, pressure, temperature and less good on the velocity components; this last quantity is indeed facing rapid variations in the boundary (Ekman) layers.

Although spectra give a good idea of the precision of the solution, they do not tell the whole story. Indeed, round-off errors also affect the solution as they are usually amplified by the resolution of linear systems (backward error) and may have a devastating effect (e.g. Valdettaro et al. 2006). In our case, round-off errors have been tested on "eigenvalues" like $\pi_{\mathrm{c}}$ and $\Lambda$ and were found to be below $5 \times 10^{-8}$.

\subsection{The virial equality}

A standard test of the accuracy of the computations of a rotating, self-gravitating fluid is the virial equality (see Ostriker \& Bodenheimer 1968; Eriguchi \& Müller 1985). This is indeed an 

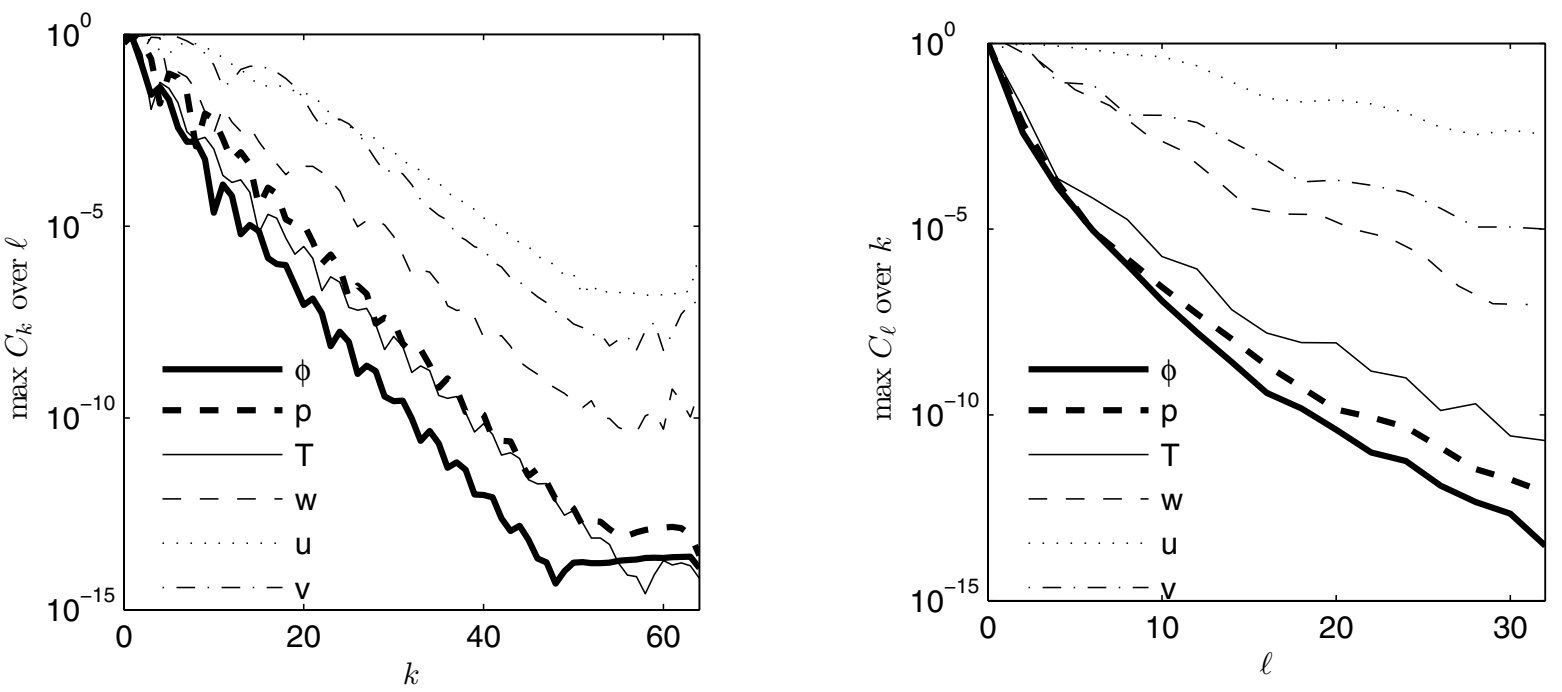

Fig. 1. Spectral convergence of the various physical quantities of the model. On left: scaled coefficient of the Chebyshev polynomial expansion (maximized over the spherical harmonic degrees); on right: the corresponding spectral coefficients of the spherical harmonics maximized over the Chebyshev coefficients. These curves have been obtained with a model where $p_{\mathrm{s}}=10^{-5}, E=10^{-8}, \Omega=0.07, \alpha=15.2, \sigma_{T}=1.5$.

integral form of the momentum equation, along with mass conservation. Since this equality has only been used with barotropic stars, we give its full expression here as is appropriate for a baroclinic star.

We first rewrite the momentum and continuity equations as

$$
\begin{aligned}
(2 \boldsymbol{\Omega} \wedge \rho \boldsymbol{u})_{i}+\rho u_{j} \partial_{j} u_{i} & =-\rho \partial_{i} \phi+\rho \Omega^{2} s\left(\boldsymbol{e}_{s}\right)_{i}+\partial_{j} \sigma_{i j} \\
\partial_{j} \rho u_{j} & =0,
\end{aligned}
$$

where $\sigma_{i j}$ stands for the stress tensor (including the pressure), namely:

$\sigma_{i j}=-P \delta_{i j}+\mu\left(\partial_{i} v_{j}+\partial_{j} v_{i}-\frac{2}{3}\left(\partial_{k} v_{k}\right) \delta_{i j}\right)$.

On the outer boundary, we imposed stress-free boundary conditions, i.e. $u_{r}=\sigma_{r \theta}=\sigma_{r \varphi}=0$. The volume integral of the scalar product of (32) with $\boldsymbol{r}$ yields the virial identity

$2 T_{\text {rel }}+I \Omega^{2}+W+3 P+I_{s}+2 \Omega L_{z}=0$,

where we introduced

$T_{\text {rel }}=\frac{1}{2} \int_{(V)} \rho u^{2} \mathrm{~d} V$

as the kinetic energy in the rotating frame,

$I=\int_{(V)} \rho r^{2} \sin ^{2} \theta \mathrm{d} V$

as the momentum of inertia,

$W=\frac{1}{2} \int_{(V)} \rho \phi \mathrm{d}^{3} \boldsymbol{r}$

as the gravitational potential energy,

$3 P=-\int_{(V)} \sigma_{i i} \mathrm{~d} V$

as the internal energy,

$I_{s}=\int_{(S)} r_{i} \sigma_{i j} \mathrm{~d} S_{j}$
Table 2. Values of the virial equality scaled by the gravitational energy for different rotation velocities.

\begin{tabular}{cc}
\hline \hline$\Omega$ & Virial \\
\hline $10^{-8}$ & $-5.4 \times 10^{-8}$ \\
0.05 & $-1.4 \times 10^{-8}$ \\
0.1 & $-1.3 \times 10^{-8}$ \\
0.15 & $1.4 \times 10^{-7}$ \\
\hline
\end{tabular}

as the surface stress and

$L_{z}=\boldsymbol{e}_{z} \cdot \int_{(V)} \boldsymbol{r} \wedge \rho \boldsymbol{u} \mathrm{d} V$

as the relative angular momentum $z$-component. The last two integrals may be vanishingly small if the background rotation contains all the angular momentum and if the boundary of the domain is near the zero pressure surface.

We give in Table 2 the value of the virial equality scaled, as usual, by the gravitational energy $W$. We have used 32 radial points, $L_{\max }=16$, Ekman number $E=10^{-5}$ and surface pressure $p_{\mathrm{s}}=10^{-3}$. For comparison, the break-up velocity for these models is $\Omega_{k} \sim 0.2$. The virial equality is verified with errors less than $10^{-7}$ thanks to spectral precision as in Bonazzola et al. (1998). These values can be compared with the $10^{-3}$ obtained by Uryu \& Eriguchi $(1994,1995)$ or $10^{-4}-10^{-5}$ by Jackson et al. (2005) resulting from the finite difference discretizations.

\subsection{Calibration with one-dimensional models}

In the dimensionless formulation, the two numbers $\Lambda$ and $\pi_{\mathrm{c}}$ are of special importance. They give the radius, the central temperature, and the central density once the mass is fixed. $\pi_{\mathrm{c}}$ is indeed a non-dimensional measure of the central pressure while $\Lambda$ of central heating. Using the expression of these numbers we note that their product

$\Lambda \pi_{\mathrm{c}}=\frac{\mathcal{R} \varepsilon_{\mathrm{c}}}{4 \pi G \rho_{\mathrm{c} \chi \mathrm{c}}}$ 
Table 3. Comparison of the results between the two-dimensional code ESTER run at zero rotation and the one-dimensional code CESAM.

\begin{tabular}{ccc}
\hline \hline & ESTER & CESAM \\
\hline Mass & 3.05 & 3.00 \\
Radius & 2.40 & 2.29 \\
Luminosity & 107. & 107. \\
$\rho_{\mathrm{c}}$ & 129 & 132 \\
$\pi_{\mathrm{c}}$ & $1.39 \times 10^{-3}$ & $1.48 \times 10^{-3}$ \\
$\Lambda$ & 232 & 219 \\
\hline
\end{tabular}

depends only on central density and central temperature; thus, its determination yields a first relation between these two quantities. The expression of the mass

$M=\rho_{\mathrm{c}} R^{3} \int_{(V)} \rho \mathrm{d} V$

combined with that of $\Lambda$ gives another relation between $T_{\mathrm{c}}$ and $\rho_{\mathrm{c}}$, thereby allowing for the determination of $R, T_{\mathrm{c}}, \rho_{\mathrm{c}}$. Actually, for convenience we prefer fixing the central temperature instead of the mass and deriving the other quantities.

As a first test of the model, we compared the results obtained for a non-rotating configuration with the results of a one-dimensional code like CESAM (Morel 1997). We show the results in Table 3 . There, we used a central temperature of $3.05 \times 10^{7}$ and a hydrogen mass fraction of $X=0.712$. The physics of the one-dimensional code was made as close to ours as possible, but surface boundary conditions, implying some superficial convection, still make noticeable differences. (The three-solar mass was chosen to minimize the effects of convection although the physics does not strictly apply in this case.) Nevertheless, the results displayed in this table show that our model is close to models computed with traditional stellar evolution codes. Thus, we have a self-gravitating fluid in our "box", close to the stellar conditions.

We do not ask for more precision at the moment since all the physics is not implemented; we leave for future work a more detailed comparison. Presently, we estimate that mass and temperature distributions are close enough to "reality" that our fluid flows are meaningful (as long as turbulence is negligible).

\section{Results}

For all the examples shown below, we have used a polar pressure of $p_{\mathrm{s}}=10^{-5}$ (scaled by central pressure), a rotation rate of $\Omega=0.07$, which is about $82 \%$ of the critical angular velocity, a central temperature of $T_{\mathrm{c}}=(b / \alpha=15.2)^{3}=1.33 \times 10^{7} \mathrm{~K}$, $\sigma_{T}=1.5$ and $\beta_{\mathrm{c}}=1$ (we neglect radiation pressure). The Ekman number was set to $10^{-8}$ and Prandtl number to zero. The mass enclosed in the container was close to one solar mass.

Table 4 summarizes the properties of the models when rotation is increased. Numerics use a radial grid with $\mathrm{Nr}=64$ points and the maximum degree of spherical harmonics is $L_{\max }=32$. Hydrogen mass fraction is $X=0.71$ and $Z=0$. Ekman number is $E=10^{-8}$. We note that the mass increases slightly, as well as the luminosity, while the radius and the central density decrease slightly. This is a consequence of our choice to keep the central temperature and the ratio of polar pressure to central pressure constant.

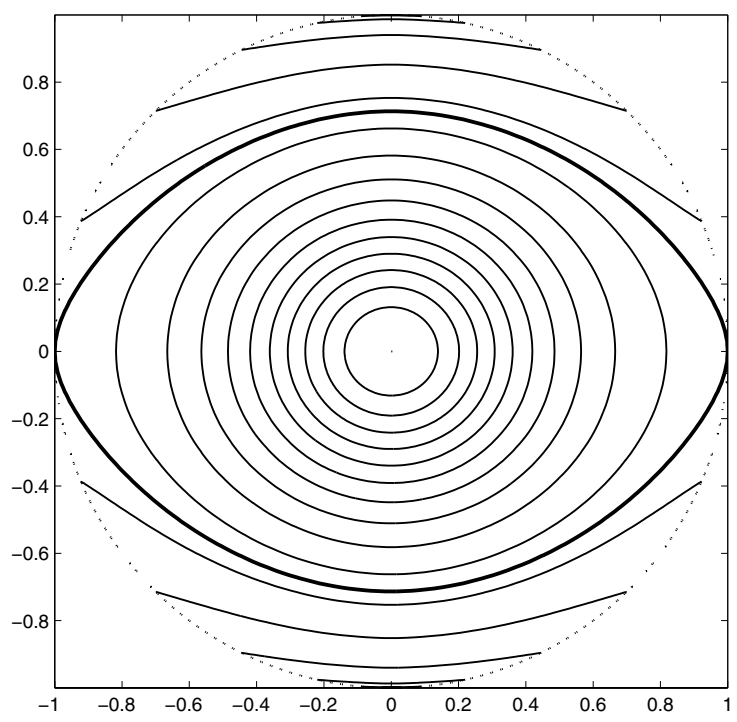

Fig. 2. Isobars for a model with $\Omega=0.07$. An equatorial Keplerian velocity for this case is reached when $\Omega_{k}=0.082$, so the star is rotating at $85 \%$ of its break-up velocity. Other parameters are standard (see text). The thick isobar underlines the last complete isobar allowed by the container.

\subsection{Internal structure}

The first view of the internal structure is given by the pressure distribution as shown in Fig. 2. We emphasize the "last isobar" with a thick line. This is the last complete isobar fitting in the container, and we use it below to appreciate the latitude variations of the flux and temperature.

Mass distribution can be appreciated by the flatness profile of the isobars, as shown in Fig. 3. It is well known that a stellar envelope with power law opacities behaves like a polytropic envelope of index $n=(\beta+3) /(\eta+1)$, which is $\sim 4.37$ in our case. Thus, for comparison, we also show the flatness of the isobar of a fully polytropic star rotating at $85 \%$ of the breakup velocity. The curves show that the polytrope is much more centrally condensed than the star, as the flatness of isobars are almost zero at the center.

We then computed the energy flux surface density at the last isobar contained in the domain (Fig. 2) and compared it to the one given by the von Zeipel model. We recall that this model assumes that the star is barotropic, so that density and temperature depend only on the effective potential (i.e. gravitational plus centrifugal); it follows that the energy flux is proportional to the local effective gravity. Figure 4 shows that the von Zeipel model overestimates the contrast of the polar and equatorial flux. Actually, the ratio of polar to equatorial flux is 3.68 for our model, while it is 6.2 for von Zeipel one, almost two times higher.

As far as temperature is concerned, we computed the variation of temperature along the last isobar. As shown in Fig. 5, the temperature drops noticeably at the equator, i.e. by a few $10^{5} \mathrm{~K}$. The temperature drop shows that isothermal surfaces are more spherical than the isobars.

Next we considered the local Brunt-Väisälä frequency which, as shown in Rieutord (2006), controls the baroclinic torque. This quantity is plotted in Fig. 6 for a rotation of $\Omega=$ 0.03 and in Fig. 7 for the usual rotation $\Omega=0.07$. Quite interestingly, we see that if rotation is fast enough, the local BruntVäisälä frequency is imaginary in the equatorial region revealing the appearance of a convection zone driven by the cool equator. 
Table 4. Parameters of our purely radiative star for various rotation rates.

\begin{tabular}{cccccccc}
\hline \hline$\Omega$ & 0.01 & 0.02 & 0.03 & 0.04 & 0.05 & 0.06 & 0.07 \\
Mass $\left(M_{\odot}\right)$ & 1.003 & 1.009 & 1.018 & 1.032 & 1.050 & 1.074 & 1.105 \\
Radius $\left(R_{\odot}\right)$ & 0.9955 & 0.9941 & 0.9918 & 0.9883 & 0.9833 & 0.9765 & 0.9674 \\
Luminosity $\left(L_{\odot}\right)$ & 0.803 & 0.805 & 0.808 & 0.811 & 0.816 & 0.822 & 0.829 \\
$\rho_{\mathrm{c}}\left(\mathrm{g} / \mathrm{cm}^{3}\right)$ & 86.5 & 86.4 & 86.3 & 86.2 & 86.0 & 85.8 & 85.6 \\
$\pi_{\mathrm{c}}$ & $5.16 \times 10^{-3}$ & $5.18 \times 10^{-3}$ & $5.21 \times 10^{-3}$ & $5.268 \times 10^{-3}$ & $5.32 \times 10^{-3}$ & $5.41 \times 10^{-3}$ & $5.53 \times 10^{-3}$ \\
$\Lambda$ & 69.2 & 69.4 & 69.0 & 68.7 & 66.9 & 65.5 & 63.7 \\
\hline
\end{tabular}

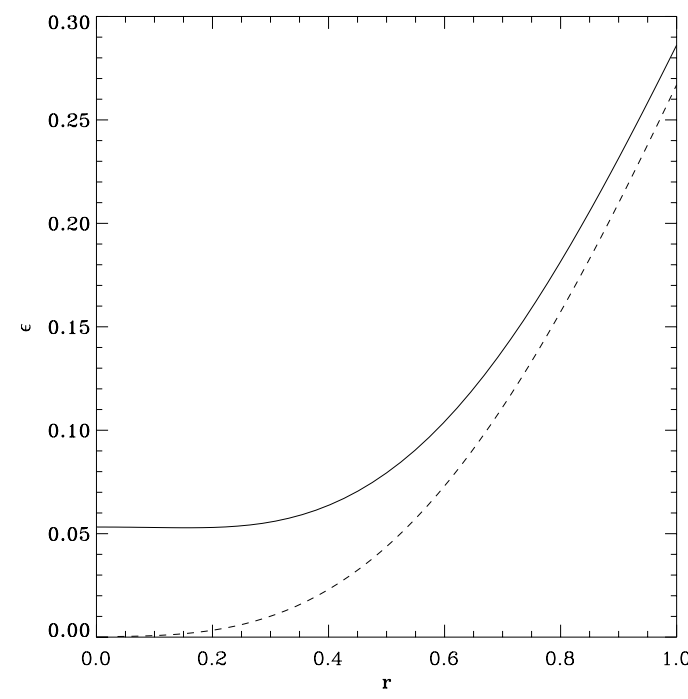

Fig. 3. Flatness $\epsilon=1-\frac{R_{\mathrm{pol}}}{R_{\mathrm{eq}}}$ of the isobars as a function of the equatorial radius for our model (solid line) and for the polytrope of index 4.37 rotating at $82 \%$ of the critical velocity. $p_{\mathrm{s}}=10^{-5}, E=10^{-8}, \Omega=0.07$, $\alpha=15.2, \sigma_{T}=1.5$.

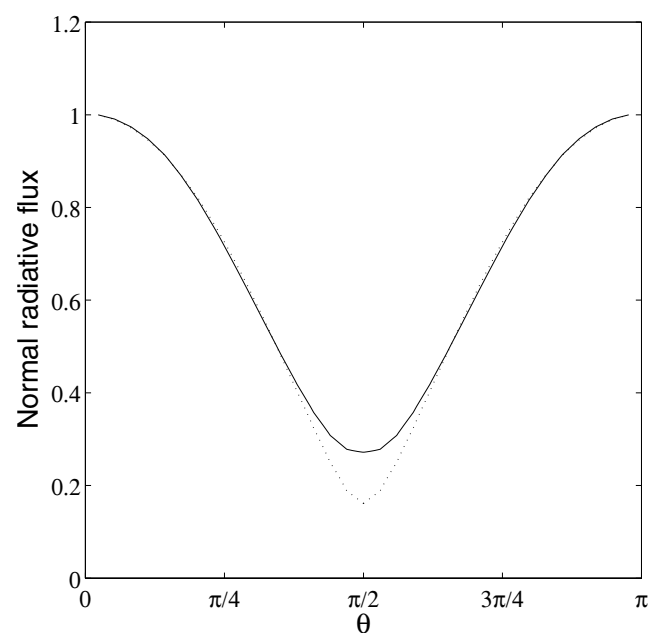

Fig. 4. Normal radiative flux at the last isobar $\left(p=p_{\text {eq }}\right)$, real values (solid line) and the von Zeipel model (dotted line). The von Zeipel model is calculated from the normal effective gravity; both curves have been scaled by their polar value.

Of course in such a region the model that we have used is no longer valid as one should take into account the entropy mixing of turbulent convection. However, we think that we show here, for the first time, the emergence of an equatorial convection zone due a fast rotation. In Fig. 8 we underline the anisotropy of the squared Brunt-Väisälä frequency distribution by comparing the poleward and equatorward profiles. This anisotropy will impact

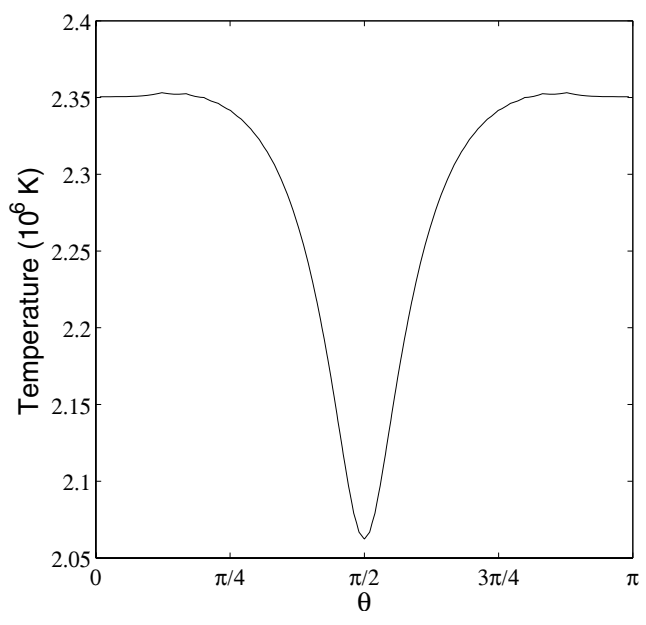

Fig. 5. Variations of temperature along the last isobar.

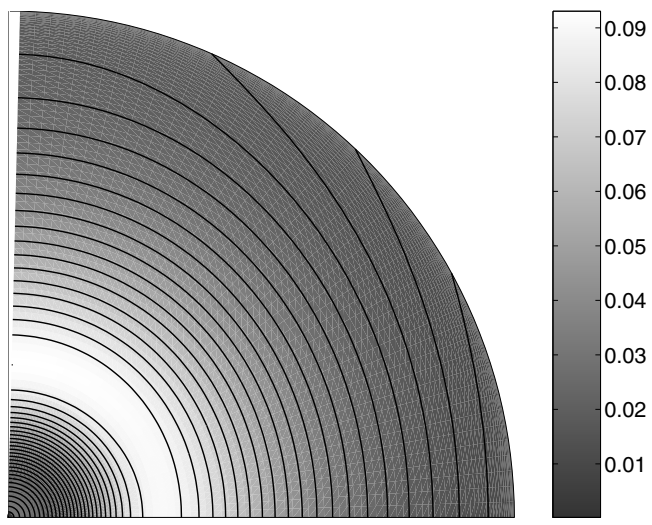

Fig. 6. Square of the Brunt-Väisälä frequency $N^{2}$ when the rotation is $\Omega=0.03$.

on the low-frequency spectrum of oscillations (gravito-inertial modes) as can be surmised from Dintrans \& Rieutord (2000).

\subsection{Differential rotation and circulation}

We now turn to the dynamical state of our radiative "star". Since we simplified the solutions by only focusing on the axisymmetric steady ones, the dynamics of the star is described by its differential rotation and meridional circulation. We give in Fig. 9 the typical shape of isorotation lines in a meridian plane. These do not change much with rotation. The main property of this baroclinic flow is that the equator rotates faster than the pole.

This shape of the differential rotation can be understood from the torque balance in the momentum equation. Indeed, neglecting nonlinear and viscous terms, the curl of the momentum 


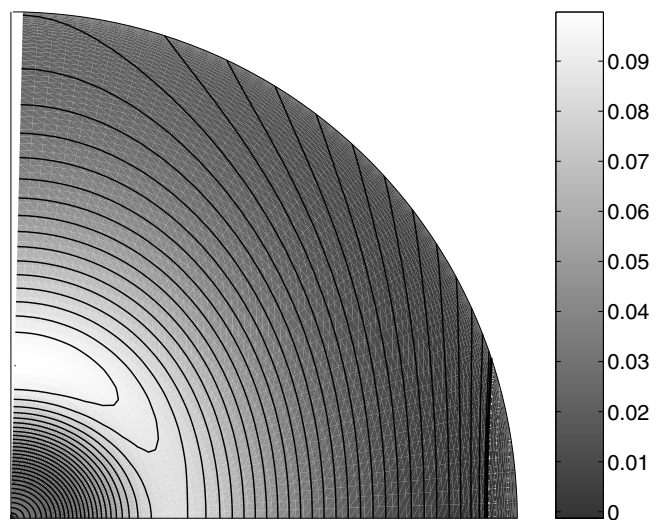

Fig. 7. Same as in Fig. 6 but with $\Omega=0.07$. Dotted lines are for negative contours, in which there is convective instability. The thick line represents the points where $N^{2}=0$.

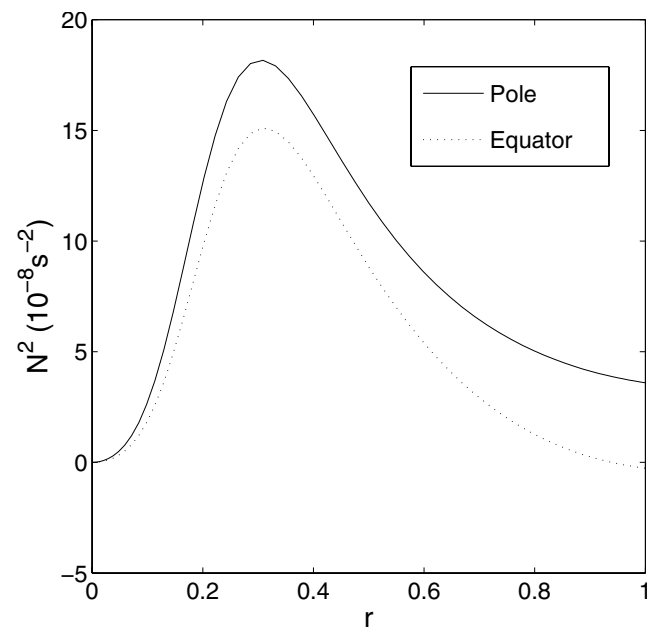

Fig. 8. Brunt-Väisälä frequency profile at pole and equator when $\Omega=0.07$.

equation in (10), together with the equation of state, yields the baroclinic balance, namely,

$\frac{\partial u_{\varphi}}{\partial z}=\pi_{\mathrm{c}}(\boldsymbol{\nabla} T \times \boldsymbol{\nabla} \ln P)_{\varphi}$

Now, if we consider the latitudinal variation of the temperature along an isobar, replacing the radial variable by the pressure, we may write $T \equiv T(P, \theta)$, and thus

$\boldsymbol{\nabla} T=\frac{\partial T}{\partial P} \boldsymbol{\nabla} P+\left(\frac{\partial T}{\partial \theta}\right)_{P} \boldsymbol{\nabla} \theta$

We can therefore rewrite the baroclinic balance as

$\frac{\partial u_{\varphi}}{\partial z}=-\frac{\pi_{\mathrm{c}}}{r}\left(\frac{\partial T}{\partial \theta}\right)_{P} \frac{\partial \ln P}{\partial r}$.

Since in any direction, pressure decreases with $r$ and isotherms are more spherical than isobars (temperature decreases on an isobar from pole to equator), we find that $\frac{\partial u_{\varphi}}{\partial z}<0$. The baroclinic torque thus makes the polar region rotate slower than the equatorial regions.

This result is (unfortunately!) at odds with the solution of the Boussinesq model devised in Rieutord (2006), where the polar rotation turned out to be faster than the equatorial one. In the Boussinesq model, the baroclinic balance leads to

$\frac{\partial u_{\varphi}}{\partial z}=N^{2} \sin \theta \cos \theta$

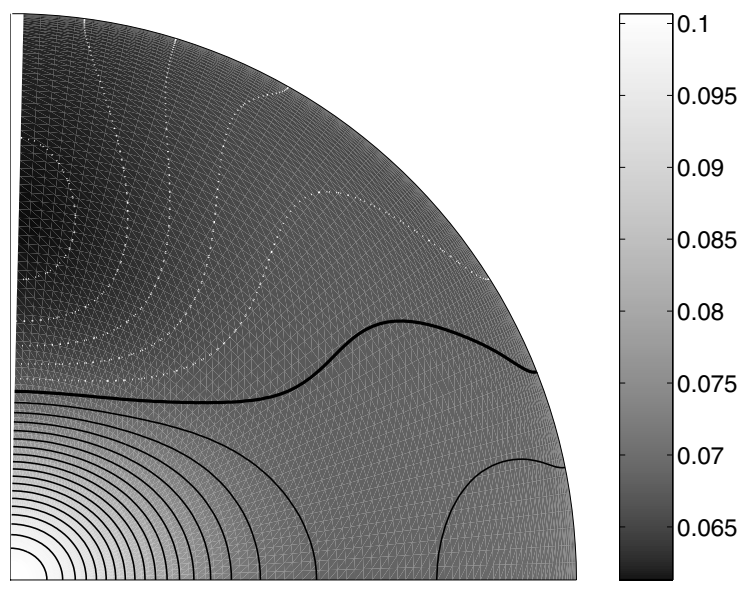

Fig. 9. Differential rotation. The thick line corresponds to $\Omega=0.07$, which is the background rotation rate. Faster and slower rotation are represented by solid black lines and dotted white lines, respectively.

which shows that rotation increases with $z$, meaning with latitude in the northern equatorial region, when the squared BruntVäisälä frequency is positive (i.e. with stable stratification).

Hence, compressibility changes the torque drastically. We traced this difference to the fact that, in the Boussinesq model, density is only a function of temperature; therefore, isochores are identical to isotherms, while isochores are strongly influence by pressure in the compressible case.

We also examined the dependence of the differential rotation on the viscosity and find that it is independent of viscosity in the asymptotic régime $E \ll 1$. This property is also shared by the Boussinesq model, which gives the explanation. As shown in Rieutord (2006), viscosity is necessary to determine the differential rotation since the inviscid case is degenerate: an arbitrary function of $r \sin \theta$ (a geostrophic flow) can always be added to $u_{\varphi}$. Viscosity lifts this degeneracy by "choosing" the geostrophic flow such that no tangential stress is exerted on the outer boundary, while the meridional flow exactly matches the Ekman pumping. As Ekman pumping and meridional flows are both proportional to viscosity, this quantity simplifies and we are left with a differential rotation independent of it.

Furthermore, an interesting property is shown in Fig. 10: except for rotations faster than $\Omega=0.03$ ( $\sim 36 \%$ of the critical angular velocity), the relative surface differential rotation keeps the same profile. Poles are $10 \%$ slower than the equator. If the shape of this profile is reachable through observations, a comparison to this shape will give interesting information on the dynamical state of the fluid (presence of turbulence, magnetic fields, etc.).

We also note from Fig. 10 that at a high rotation rate, a kind of equatorial jet develops between latitudes $\pm 20^{\circ}$. If confirmed by more realistic models, such a jet may play an important role in mass loss for stars rotating close to the breakup limit.

The case of meridional circulation, displayed in Fig. 11, shows three cells of circulation. This feature of the meridional flow is controlled by the distribution of the Brunt-Väisälä frequency and the Prandtl number as shown in the Boussinesq case. As far as the turnover time associated with this circulation is concerned, we refer to the discussion in Rieutord (2006); indeed, except the profile of density, there is no significant change, because the turnover time remains controlled by viscosity (probably turbulent) and the Prandtl number.

Just as in the Boussinesq case questions of stability would require a full study. As in Rieutord (2006), we nevertheless take a look at the case of axisymmetric barotropic stability. We thus 


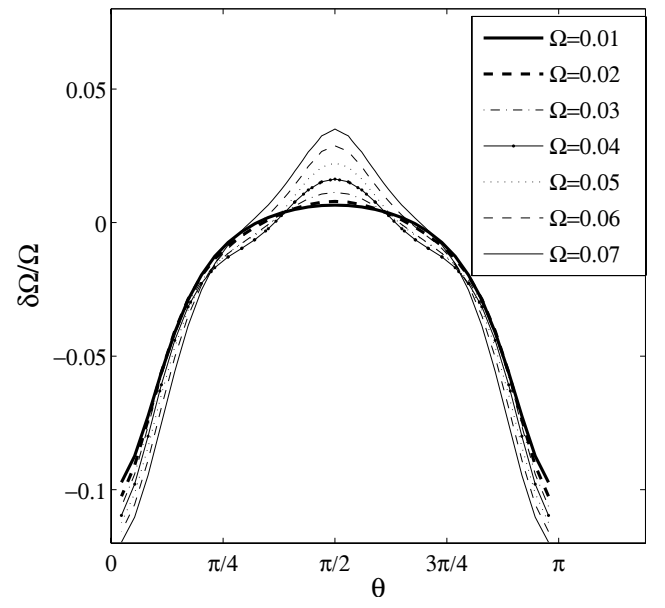

Fig. 10. Relative differential rotation on the last isobar.

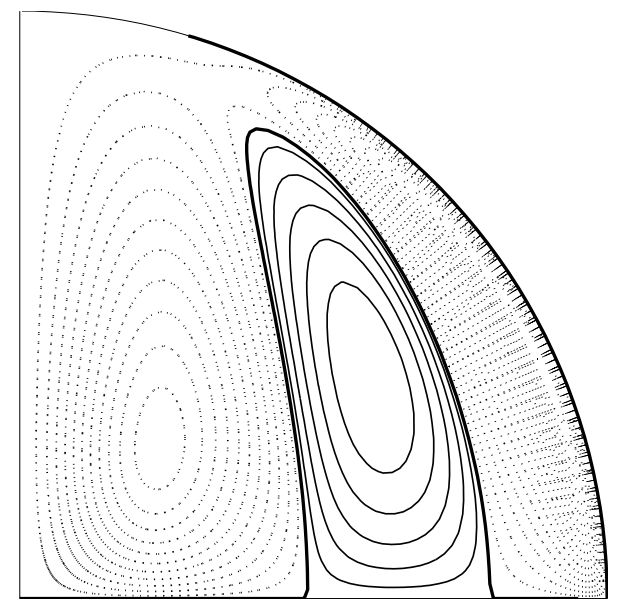

Fig. 11. Meridional circulation. Solid lines represent counterclockwise circulation and dotted lines clockwise circulation. $p_{\mathrm{s}}=10^{-5}, E=10^{-8}$, $\Omega=0.07, \alpha=15.2, \sigma_{T}=1.5$.

compute the angular momentum distribution along an isentropic surface; more precisely, we verify that, along such a surface, a fluid parcel has an increasing angular momentum when its position gets farther from the rotation axis. This is a necessary condition for axisymmetric stability, and Fig. 12 shows that this is indeed the case.

\subsection{About the effects of the container}

Finally, one may wonder what the role of the box containing our "star" is. Obviously, as far as the hydrostatics is concerned, the main effect of this box is to reduce the radius (the container cuts out the envelope). One may also wonder about the consequences of the conflicting spherical geometry of the box and the spheroidal shape of the mass distribution, on the latitudinal flux variations. We find that our model gives a milder ratio of the polar to equatorial flux than the von Zeipel model; actually, this difference may be reduced when the container is eliminated because its spherical geometry may soften the latitudinal variations.

We also conjecture that the effects of the container on dynamics are fairly weak. Indeed, we note that, while changing $\Omega$ and thus the shape of isobars and isotherms, the form of the differential rotation remains very similar. As the flow comes essentially from the mismatch of isobars and isotherms, which both

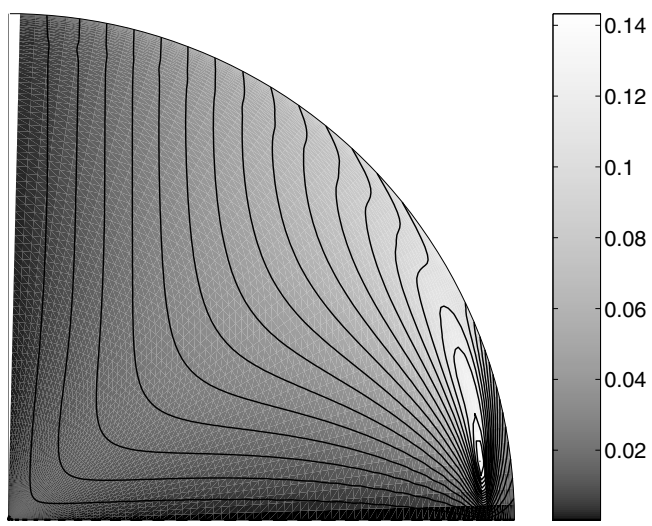

Fig. 12. Directional derivative of $L_{z}$ along equal entropy lines, i.e. ( $\boldsymbol{n}$. $\boldsymbol{\nabla}) L_{z}$ where $\boldsymbol{n}$ is a unit vector tangential to the isentropy surface and directed towards the equator.

change with respect to the sphere as $\Omega$ varies, its invariance at low $\Omega$ pleads for a robust result, independent of the walls of the container.

\section{Conclusions}

In this paper we presented a physically self-consistent model of a rotating self-gravitating perfect gas heated by nuclear reactions and enclosed in a container with a constant absorption coefficient radiating like a black body; heat transport in the gas is purely radiative, insured by a power law opacity.

This is a simplified model for a completely radiative star that rotates at a significant fraction of the breakup angular velocity. We thus generalized the approach of Rieutord (2006) by taking more realistic properties of a stellar plasma into account. Such a fluid is never in hydrostatic equilibrium and a baroclinic flow develops. Taking viscosity into account, we computed such a flow in its steady state, namely the differential rotation and the meridional circulation. Interestingly enough, the results show that equatorial regions are rotating faster than polar ones and, provided that rotation is less than $\sim 36 \%$ of the breakup velocity, the surface profile $\delta \Omega / \Omega$ is independent of both viscosity and rotation rate.

Although our model is still preliminary, we had a look at the anisotropy of the emitted flux to find that the pole/equator ratio is less contrasted than what is predicted by the von Zeipel model. We also showed that, when rotation is fast enough, equatorial regions become convectively unstable.

In parallel to constructing this model, we tested - for the first time to our knowledge - the use of spectral methods in the stellar structure computations. Our first results are promising in terms of precision and robustness, since we recover the precision of a finite difference type model with ten times less grid points. Such a gain in efficiency is necessary for all future computations of the evolution of a rotating star with a two-dimensional model.

The next step is of course to confirm these results using a model with coordinates adapted to the spheroidal shape of the isobars. In this case boundary conditions can be cleanly applied, and the surface pressure can be decreased to very low values adapted to matching an atmosphere model. Of course, including modeled convection zones is also a necessary step for the completeness of models to be used in the interpretation of observations.

Acknowledgements. We thank Bernard Pichon for providing us with 1D models from the CESAM code and for many helpful discussions. This work is part of the 
ESTER project aimed at modeling stars in two dimensions. It is supported by the Programme National de Physique Stellaire along with the Action Spécifique pour la Simulation Numérique en Astrophysique. FE thanks the CNRS postdoctoral program for its support during his stay at the LATT in Toulouse.

\section{References}

Aufdenberg, J. P., Mérand, A., Coudé du Foresto, V., et al. 2006, ApJ, 645, 664 Bonazzola, S., Gourgoulhon, E., \& Marck, J.-A. 1998, Phys. Rev. D, 58, 104020 Bonazzola, S., Gourgoulhon, E., \& Marck, J.-A. 1999, J. Comput. Appl. Math., 109,433

Canuto, C., Hussaini, M. Y., Quarteroni, A., \& Zang, T. A. 2006, Spectral Methods: Fundamentals in Single Domains (Springer Verlag)

Christensen-Dalsgaard, J., \& Reiter, J. 1995, in GONG 1994. Helio- and AstroSeismology from the Earth and Space, ed. R. K. Ulrich, E. J. Rhodes, Jr., \& W. Dappen, ASP Conf. Ser., 76, 136

Dintrans, B., \& Rieutord, M. 2000, A\&A, 354, 86

Domiciano de Souza, A., Vakili, F., Jankov, S., Janot-Pacheco, E., \& Abe, L. 2002, A\&A, 393, 345

Domiciano de Souza, A., Kervella, P., Jankov, S., et al. 2005, A\&A, 442, 567

Eriguchi, Y., \& Müller, E. 1985, A\&A, 146, 260

Fornberg, B. 1998, A practical guide to pseudospectral methods (Cambridge University Press)
Grandclément, P. 2006, in Stellar fluid dynamics and numerical simulations: from the Sun to neutron stars, ed. M. Rieutord, \& B. Dubrulle (EDP Sciences), 153

Jackson, S., MacGregor, K. B., \& Skumanich, A. 2005, ApJS, 156, 245

Kervella, P., \& Domiciano de Souza, A. 2006, A\&A, 453, 1059

McAlister, H. A., ten Brummelaar, T. A., Gies, D. R., et al. 2005, ApJ, 628, 439

Morel, P. 1997, A\&AS, 124, 597

Ostriker, J. P., \& Bodenheimer, P. 1968, ApJ, 151, 1089

Peterson, D., Hummel, C., Pauls, T., et al. 2006a, ApJ, 636, 1087

Peterson, D. M., Hummel, C. A., Pauls, T. A., et al. 2006b, Nature, 440, 896

Peyret, R. 2002, Spectral methods for incompressible viscous flow (New-York: Springer)

Reese, D., Lignières, F., \& Rieutord, M. 2006, A\&A, 455, 621

Rieutord, M. 1987, Geophys. Astrophys. Fluid Dyn., 39, 163

Rieutord, M. 2006, A\&A, 451, 1025

Roxburgh, I. W. 2004, A\&A, 428, 171

Uryu, K., \& Eriguchi, Y. 1994, MNRAS, 269, 24

Uryu, K., \& Eriguchi, Y. 1995, MNRAS, 277, 1411

Valdettaro, L., Rieutord, M., Braconnier, T., \& Fraysse, V. 2006, J. Comput. Appl. Math., 205, 382, [physics/0604219]

Zahn, J.-P. 1992, A\&A, 265, 115

Zorec, J., Domiciano de Souza, A., Frémat, Y., \& Vakili, F. 2005, in SF2A2005: Semaine de l'Astrophysique Francaise, ed. F. Casoli, T. Contini, J. M. Hameury, \& L. Pagani, 363 